\title{
How Polydopamine Modulates Biological Responses to PTFE Prostheses
}

\author{
Isabelle Talon ${ }^{1,2}$, Anne Schneider ${ }^{1,2}$, Eric Mathieu', Bernard Senger ${ }^{1}$, \\ Benoit Frisch $^{3}$, Cendrine Seguin ${ }^{3}$, Vincent Ball1, Joseph Hemmerlé1
}

\author{
${ }^{1}$ National Institute of Health and Medical Research, INSERM UMR_S 1121, 11 rue Humann, Strasbourg, France \\ ${ }^{2}$ Pediatric surgery Department, University Hospital of Strasbourg, Avenue de Moliere, Strasbourg, France \\ ${ }^{3}$ National Center of Scientific Research, CNRS UMR_7199, UMR_7199, University of Pharmacy of Strasbourg, 74 Route du Rhin-CS \\ 60024, Illkirch Cedex, France \\ Email: *isabelle.TALON@chru-strasbourg.fr
}

How to cite this paper: Talon, I., Schneider, A., Mathieu, E., Senger, B., Frisch, B., Seguin, C., Ball, V. and Hemmerlé, J. (2019) How Polydopamine Modulates Biological Responses to PTFE Prostheses. Materials Sciences and Applications, 10, 377-392.

https://doi.org/10.4236/msa.2019.105028

Received: January 27, 2019

Accepted: May 4, 2019

Published: May 7, 2019

Copyright $\odot 2019$ by author(s) and Scientific Research Publishing Inc. This work is licensed under the Creative Commons Attribution International License (CC BY 4.0).

http://creativecommons.org/licenses/by/4.0/

\section{c) (i) Open Access}

\begin{abstract}
Diaphragm repair after congenital diaphragmatic hernia is associated with hernia recurrence due to prosthesis failure. Expanded polytetrafluoroethylene (e-PTFE), a synthetic non-degradable biomaterial, is currently used for those diaphragmatic defect repairs. The drawback of e-PTFE is its poor wettability that leads to coating difficulties, bonding that could favor implant integration. However, polydopamine (PDA) can be deposited as well on organic as on inorganic substrates. Therefore, we assessed the biological responses of a clinically used e-PTFE biomaterial treated with PDA in two different manners: one impregnated with PDA and the other coated with a one side PDA film. Mechanical properties of the raw e-PTFE, the PDA soaked biomaterial and the PDA coated surface were characterized by colloidal probe atomic force microscopy. Behaviors of primary human fibroblasts and Wharton's jelly stem cells were investigated by electron microscopy. Findings reveal that the mechanical properties at the microscopic scale are not modified by the PDA treatments. Cells spread onto both PDA functionalized substrates. In addition, microscopic observations disclose numerous focal cell contacts, evidencing cell attachment, and cytoplasmic projections particularly with the nanoscale PDA coating. Results clearly suggest that PDA in general but above all the PDA coating enhance cellular colonization of the implant material.
\end{abstract}

\section{Keywords}

Biomaterials, Expanded Polytetrafluoroethylene, Polydopamine, Functionalization, Electron Microscopy

\section{Introduction}

The diaphragm that forms a physical barrier between the thoracic and the ab- 
dominal cavities is also the major muscle of the respiratory system. Congenital diaphragmatic hernia $(\mathrm{CDH})$ is a malformation of that partition muscle. Its incidence rate oscillates around 1 per 2500 births [1]. CDH requires surgical treatment for closing the diaphragm defect. It is well known that cell adhesion and proliferation onto and/or within biomaterials depend on surface characteristics as, for instance, chemistry, wettability and topography. Thus, surface features of implant materials are of utmost importance for guiding cellular response, behavior and organization [2] [3]. To mimic the biological and biomechanical features of the native diaphragm, biomaterials for $\mathrm{CDH}$ repair should favor muscle cell in growth and target the formation of functional muscle tissue [4].

The ideal biomaterial for diaphragm repair doesn't exist yet. It should be biocompatible, long-lasting and elastic. It is still quite difficult to find a biomaterial that fits with all these requirements. Therefore, non-elastic patches of expanded polytetrafluoroethylene (e-PTFE), a synthetic non-degradable biomaterial, are currently used for the repair of diaphragm defects [4].

Although the properties of PTFE are of high interest in medical applications, including arterial grafts and reconstructive facial surgery, the polymer produced by polymerization of tetrafluoroethylene (known by trademarks such as Teflon ${ }^{\circledR}$ and Gore-Tex ${ }^{\circledR}$ ) is inert to most chemicals [5]. That is why PTFE is used to make non-stick cooking pans. The chemical stability of PTFE is related to the strength of the fluorine-carbon bond. When stretched, PTFE forms a strong porous material called expanded PTFE (e-PTFE) [6].

The poor wettability of PTFE leads to bonding problems for coatings. Chemical functionalization is needed to fix bioactive molecules to its surface. Gas plasma surface modification, for instance, has been used to benefit from the bulk properties of PTFE [4].

Polydopamine (PDA) was used for the first time in 2007 for the coating of surfaces. The undeniable advantage of PDA is that it can coat as well all organic and all inorganic substrates [7] [8]. The chemical structure of PDA, which incorporates many functional groups such as catechols, quinones, semi quinones and amines, is of the highest importance for post-functionalization. A PDA undercoat can serve as a starting layer for further covalent modification [7].

In the present study, we assessed the biological responses to a clinically used e-PTFE biomaterial treated with PDA in two different manners, providing two different implant materials. Treatment of a biomaterial by dipping it into a dopamine solution allows numerous secondary functionalization methods. Despite its evident interest in the medical field, the dipping of a porous implant material, as e-PTFE, in a PDA solution produces a biomaterial sheet with equal properties on each side. With the goal of modifying exclusively one side of the porous e-PTFE material, we used the Langmuir-Schaeffer deposition technique [8]. This particular approach is of high concern in the case of $\mathrm{CDH}$ implants, where the expected host responses have to be different at each side: the surface facing the abdominal cavity should prevent tissue adhesions, thus avoiding bowel occlusion. 
Here we investigated the behaviors of primary dermal human fibroblasts and Wharton's jelly stem cells on the raw biomaterial, on e-PTFE soaked in a PDA solution and on e-PTFE coated at one side only with a PDA film by using scanning electron microscopy (SEM), transmission electron microscopy (TEM) and atomic force microscopy (AFM). We also evaluate mitochondrial activity, cell viability and inflammatory cytokine production in immune cells when in contact with the raw biomaterial and biomaterial treated by PDA.

\section{Materials and Methods}

\subsection{Polydopamine Functionalization of the E-PTFE Material}

Polydopamine films at the air/water interface then transferred on one side of e-PTFE.

Such conditions will be denoted as e-PTFE_f (for film) in the following.

Dopamine hydrochloride was purchased from Sigma-Aldrich (Saint Louis, $\mathrm{MO}$ ). Dopamine hydrochloride at $2 \mathrm{mg} \cdot \mathrm{mL}^{-1}$ was dissolved in Tris buffer at 50 $\mathrm{mM}(\mathrm{pH}=8.5)$ with oxygen as the oxidant. Tris(hydroxymethyl) aminomethane was obtained from Euromedex (Souffelweyersheim, France). All the solutions were prepared from ultra pure water (Milli Qplus system, $\rho=18.2 \mathrm{M} \Omega \cdot \mathrm{cm}$, Merck-Millipore). Note that dopamine was dissolved in the Tris buffer just before the film deposition experiment at either the air/water or the solid/liquid interface.

The solutions were left unstirred for 3 hours which is the optimal reaction time [8]. Indeed, due to water evaporation and because PDA films stick on the wall of the glass ware, high shear forces appear and lead to the formation of cracks in the deposited PDA film which, in turn, weaken the coating at longer reaction times. The creation of cracks was observed in situ with a Leica DM 4000 $\mathrm{M}$ microscope in the reflection mode under bright field illumination. The films were then transferred from the Air /Water interface to the e-PTFE with the Langmuir-Schaeffer technique at a displacement speed of the horizontal substrate toward the film of $0.01 \mathrm{~cm} \cdot \mathrm{s}^{-1}$ and in the opposite direction with a speed of $0.02 \mathrm{~cm} \cdot \mathrm{s}^{-1}$. The contact time of the substrate with the PDA film formed at the air/water interface was of one minute before moving the substrate in the vertical direction. The films were then rinsed thoroughly with pure water and dried under a nitrogen stream. The substrates used for this study were $1 \mathrm{~mm}$ thick squares of $1 \mathrm{~cm}^{2}$ of e-PTFE and others (Dualmesh ${ }^{\circledR}$ W.L. Gore and Associates, Flagstaff, AZ).

Polydopamine deposition at the solid/liquid interface by dipping e-PTFE in a PDA solution

Such conditions will be called e-PTFE_i (for impregnated) in the following.

Dopamine hydrochloride at $2 \mathrm{mg} \cdot \mathrm{mL}^{-1}$ was dissolved in Tris buffer at $50 \mathrm{mM}$ $(\mathrm{pH}=8.5)$ with oxygen as oxidant. Samples of e-PTFE were dipped in the dopamine solution for 3 hours. They were then rinsed thoroughly with pure water and dried with a nitrogen jet. In this way, both sides of the substrate are coated with PDA. 


\subsection{Contact Angle Measurements}

For the static contact angle measurements, $3 \mu \mathrm{L}$ of distilled water $(\rho=18.2$ $\mathrm{M} \Omega \cdot \mathrm{cm}$ ) was dropped on the air side surface of the PTFE membrane at room temperature, and the contact angle was measured after $10 \mathrm{sec}$. The given values correspond the average \pm one standard deviation over $n=3$ measurements. A OneAttension goniometer fitted with the OneAttension 3.1 software and image acquisition software was used for those measurements (Biolin Scientific).

\subsection{Cellular Evaluation}

Three different conditions and one glass-control were studied: non-treated e-PTFE, e-PTFE_f and e-PTFE_i. The pretreated substrates were autoclave-sterilized for cell culture.

Primary dermal human fibroblasts were harvested from human preputial connective tissue. The method used is the explant culture: small fragments of connective tissue (about $2 \mathrm{~mm}^{3}$ ) were placed in a plastic flask with $2 \mathrm{~mL}$ DMEM (Dulbecco's Modified Eagle Medium) with 20\% FBS (Fetal Bovine Serum), 1\% non-essential amino acids and 1\% Penicillin/Streptomycin, in a tissue culture incubator. Growth media was initially changed after 5 days, and then 3 times a week until confluence.

For the seeding, primary human fibroblasts were used between passages 2 and 4. They were detached from the culture flask by treatment with trypsin/EDTA and seeded onto the pretreated prosthetic specimens at a concentration of $1 \times$ $10^{4}$ cells/well. They were cultured in DMEM, supplemented with $10 \%$ FBS, $1 \%$ non-essential amino acids and $1 \%$ Penicillin/Streptomycin, at $37^{\circ} \mathrm{C}$ in a $5 \% \mathrm{CO}_{2}$, and humidified atmosphere. DMEM was changed every $48 \mathrm{~h}$ and the culture was stopped after 6 days using primary fixation with $4 \%$ glutaraldehyde and $1 \%$ paraformaldehyde.

Wharton's jelly stem cells (WJ-SC) were enzymatically isolated from human umbilical cords obtained after full-term births. WJ-SC were amplified at a density of $3 \times 10^{3}$ cells $/ \mathrm{cm}^{2}$ in $\alpha$-MEM (Minimum Essential Medium Eagle Alpha Modification) culture medium supplemented with $10 \%$ decomplemented FBS, $1 \%$ Penicillin/Streptomycin/Amphotericin B and 1\% $\operatorname{Glutamax}^{\circledR}(v / v$, Gibco) and maintained in a humidified atmosphere of $5 \% \mathrm{CO}_{2}$ at $37^{\circ} \mathrm{C}$ with a medium change every 2 days.

WJ-SC were used at the $3^{\text {rd }}$ passage and seeded onto the prepared prosthetic specimens at a concentration of $10^{4} \mathrm{cells} / \mathrm{cm}^{2}$. Cells were maintained in $\alpha$-MEM supplemented with $10 \%$ decomplemented FBS, 1\% Penicillin/Streptomycin/ Amphotericin B and $1 \%$ Glutamax $^{\circledR}$. Cultures were stopped after 2 days using primary fixation with $4 \%$ glutaraldehyde and $1 \%$ paraformaldehyde.

Cellular experiments were approved ethically and methodologically by the local Research Institution (Registration number DC-2015-2364). Informed signed consent was obtained from the patients. 


\subsection{Electron Microscopy}

\section{Sample processing}

e-PTFE samples after cell culture were fixed in $4 \%$ glutaraldehyde and $1 \%$ paraformaldehyde buffered in sodium cacodylate $0.1 \mathrm{M}$ at $\mathrm{pH}=7.4$. After 3 washes of $10 \mathrm{~min}$ in the same buffer, the samples were post-fixed with $1 \%$ osmic acid in a $0.1 \mathrm{M}$ sodium cacodylate solution for $45 \mathrm{~min}$. These chemicals were purchased from Euromedex (Souffelweyersheim, France). Then, the samples were dehydrated in graded series of ethanol for $10 \mathrm{~min}$ each. In order to increase resin impregnation, we used Spurr resin (Sigma Aldrich, Saint Quentin Fallavier, France) that has a lower viscosity than Epon resin. The composition of Spurr mix used (adapted for this sample) was the following: $5.90 \mathrm{~g}$ of NSA (Nonenyl Succinic anhydride), $4.10 \mathrm{~g}$ of ERL4221 (cycloaliphatic epoxide resin), $1.59 \mathrm{~g}$ of DER 736 (Diglycidyl ether of polypropyleneglycol) and $0.1 \mathrm{~g}$ DMAE (Dimethylaminoethanol) as an accelerator.

e-PTFE samples were transferred in 1 vol Spurr resin/2 vol absolute ethanol for $30 \mathrm{~min}, 1 \mathrm{vol}$ Spurr resin/1 vol absolute ethanol for $1 \mathrm{~h}$, and finally in 100\% Spurr resin for $2 \mathrm{~h}$. After one night in 100\% Spurr resin, the e-PTFE samples were embedded in freshly prepared resin and polymerized for $48 \mathrm{~h}$ at $60^{\circ} \mathrm{C}$.

To be able to perform ultra-thin sections, we reduced the size of the e-PTFE samples as much as possible and then re-embedded the trimmed specimens using the above-mentioned resin. Ultra-thin sections were performed by using an automatic ultra-microtome Reichert Jung Ultracut E (Leica Microsystems, Nanterre, France) equipped with a diamond knife. Ultrathin sections were gently heated at $45^{\circ} \mathrm{C}$ during $3 \mathrm{~min}$ by putting the diamond knife on a warm plate (Lab-Line Instruments Inc., Ill, USA) to eliminate possible folds.

Ultra-thin sections were collected on 100 mesh carbon-coated formvar-covered TEM grids and stained in a 5\% uranyl acetate solution (Euromedex, Souffelweyersheim, France) for $20 \mathrm{~min}$. After rinsing in 50\% ethanol, grids were double-stained in a $4 \%$ lead citrate solution for $10 \mathrm{~min}$ (Euromedex, Souffelweyersheim, France).

\section{Electron microscopy}

Transmission electron microscopy was performed by using a Philips EM 208 instrument (FEI Company, Eindhoven, The Netherlands) operating with an accelerating voltage of the electrons of $70 \mathrm{kV}$. Micrographs were recorded on Kodak SO163 films and scanned with an Epson Perfection scan V500 PRO (Epson France, Levallois-Perret, France).

Scanning electron microscopy was performed on samples that were fixed during $4 \mathrm{~h}$ in the same aldehyde solution employed for TEM preparations and then post-fixed with osmium tetroxide for $1 \mathrm{~h}$. The fixed specimens were dehydrated in a graded ethanol series, critical point dried and gold-palladium coated. Observations were carried out by using a Hitachi TM-1000 (Hitachi, Tokyo, Japan) tabletop scanning electron microscope and a Quanta-250 (FEI Company, Eindhoven, The Netherlands) environmental scanning electron microscope (ESEM). 


\section{Atomic Force microscopy}

AFM experiments were realized by using a Bioscope Catalyst apparatus (Bruker Nano Surfaces Division, Santa Barbara, CA USA).

Stiffness measurements were performed at different areas at room temperature. The MIRO ${ }^{\mathrm{TM}}$ (Microscope Image Registration and Overlay) software was used to select the areas of interest and target discrete points for force measurements. Specimens were indented with borosilicate beads, $10 \mu \mathrm{m}$ in diameter, mounted on silicon nitride AFM probes (Novascan Technologies, Ames, IA USA) having a nominal spring constant value of $0.06 \mathrm{~N} / \mathrm{m}$. The cantilever spring constant was determined with the thermal fluctuation method [9]. Experiments were carried out at constant approach and withdrawal velocities. To characterize the micro-scale stiffness of the samples, the recorded force curves were processed using the commercial NanoScope Analysis software (Bruker Corporation, Santa Barbara, CA USA), which offers a full suite of force curve analysis tools. The Young's moduli of the surfaces were obtained by fitting the Hertz law [10] to the approach curve from the contact point up to a point corresponding to an indentation not exceeding a few percent of the radius of the colloidal probe.

\subsection{Measurement of Mitochondrial Activity and Inflammatory Cytokine Production in Immune Cells}

To assess the impact of functionalized e-PTFE on immune cells, we use in a first attempt murin monocytes/macrophages.

Culture media and supplement are all from Sigma-Aldrich (Saint Louis, MO). RAW 264.7 cells were a gift from S. Lazereg (ICSN, Gif sur Yvette, France). RAW264.7 macrophages were cultured in DMEM AQ Media with high glucose $(4500 \mathrm{mg} / \mathrm{L})$ supplemented with $5 \%$ heat-inactivated fetal bovine serum, penicillin $(100 \mathrm{U} / \mathrm{mL})$, and streptomycin $(100 \mu \mathrm{g} / \mathrm{mL})$. The cells were maintained in a humidified incubator containing $5 \% \mathrm{CO}_{2}$ at $37^{\circ} \mathrm{C}$. For the experiments, cell concentration was adjusted to $10^{6}$ cells $/ \mathrm{mL}$ and $10^{5}$ cells/well were seeded in 96 well plates. As macrophage activation positive control, LPS at $500 \mathrm{ng} / \mathrm{mL}$ was used, and as positive dead cell control, 5\% DMSO was used. Samples of e-PTFE were kept at the bottom of the wells by sterile plastic rings. $24 \mathrm{~h}$ following treatment, mitochondrial activity was assessed using CellTiter $96^{\circledR}$ AQueous One Solution Cell Proliferation Assay (Promega, Madison, WI). Briefly, after supernatant removal, $100 \mu \mathrm{L}$ PBS1X and $20 \mu \mathrm{L}$ MTS solution was added per well. Cells were incubated at $37^{\circ} \mathrm{C}$ for $40 \mathrm{~min}$ and absorbance was measured at 490 and 700 $\mathrm{nm}$ on a SAFAS spectrophotometer (SAFAS, Monaco). Cell viability was determined as the optical density ratio between sample and control cells. To determine the effects of PDA on the production of inflammatory cytokines (i.e., TNF- $\alpha$ ), cells were cultured and treated as described. Supernatants collected from wells of a 96-well plate were assayed using specific enzyme-linked immunosorbent assay (ELISA). Purified anti-mouse TNF- $\alpha$ antibody clone 1F3F3D4 and biotinylated anti-mouse TNF- $\alpha$ antibody clone XT3/XT22 were purchased from eBiosciences/thermoFischer Scientific (Waltham, MA, USA). Avidin HRP 
was from Jackson (West Grove, PA, USA).

\subsection{Statistical Analysis}

Data analysis was performed using the Kruskal-Wallis one-way analysis of variance on ranks by means of the computer software package SigmaStat ${ }^{\circledR}$ (SPSS Inc., Chicago, IL, USA).

\section{Results}

SEM observations of the raw e-PTFE biomaterial (Figure 1(a)), the e-PTFE_i

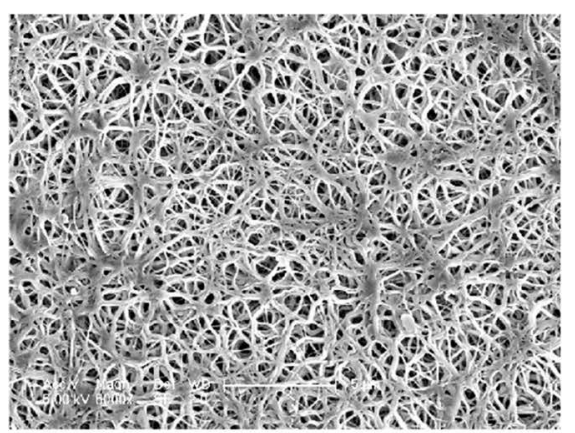

(a)

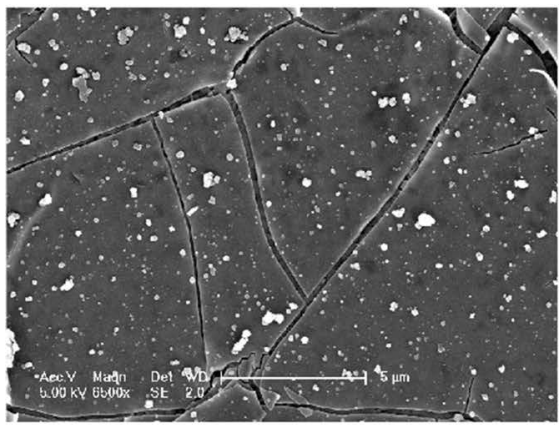

(c)

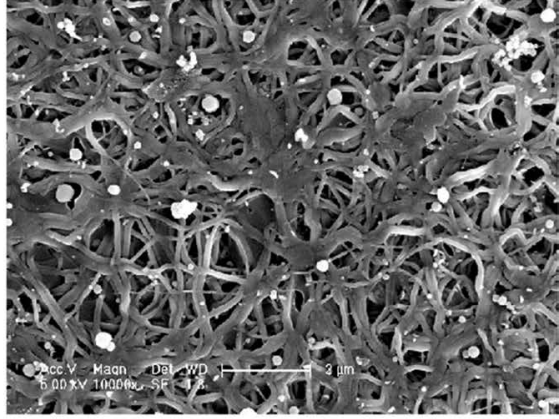

(b)

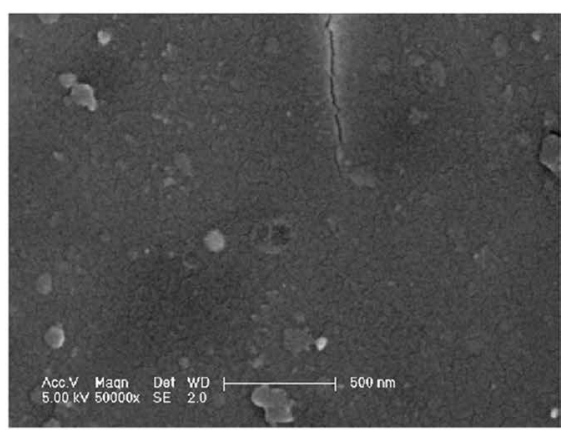

(d)

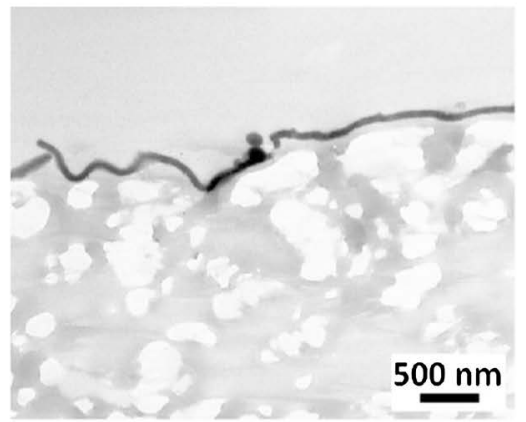

(e)

Figure 1. (a) SEM micrograph of the porous e-PTFE biomaterial. Scale bar $=5 \mu \mathrm{m}$. (b) SEM micrograph of the e-PTFE_i surface. Scale bar $=2 \mu \mathrm{m}$. (c) SEM micrograph of the e-PTFE_f surface. Cracks show the e-PTFE substrate under the PDA film. Scale bar $=5$ $\mu \mathrm{m}$. (d) High magnification SEM micrograph revealing the granular aspect of the polydopamine layer deposited by the Langmuir-Schaeffer method. Scale bar $=0.5 \mu \mathrm{m}$. (e) TEM micrograph showing a polydopamine film lining the biomaterial's surface from an e-PTFE_f material. Scale bar $=500 \mathrm{~nm}$. 
(Figure 1(b)) and the e-PTFE_f surface (Figure 1(c)) revealed quite different surface features. The raw implant material exhibited a highly fibrillar texture providing micrometric porosity (Figure $1(\mathrm{a})$ ). The e-PTFE_i kept the fibrillar aspect of the e-PTFE, but exhibited a thickening of the individual PTFE fibers (Figure 1(b)). In contrast, e-PTFE_f showed a rather flat surface (Figure 1(c)). At higher magnification, it appeared that the e-PTFE_f (Figure 1(d)) is of granular nature. The cracks of the thin PDA layer disclosed fibrils of the underlying e-PTFE material. The sparse white spots on the PDA treated samples (Figure $1(\mathrm{~b})$, Figure 1(c)) correspond to PDA aggregates. Both the soaked and the coated e-PTFE were also visualized by AFM. AFM results demonstrated that the individual PTFE fibrils at the surface of the e-PTFE_i are decorated with tiny PDA particles measuring between 35 and $60 \mathrm{~nm}$, whereas the surface of e-PTFE_f is covered by a continuous film of similarly sized PDA grains. TEM observation of a cross-section of the PDA coating deposited by the Langmuir-Schaeffer method revealed that the biomaterial is covered by a $80 \mathrm{~nm}$ thick PDA film (Figure 1(e)).

The mechanical properties of the raw e-PTFE (Figure 2(a), Figure 2(b)), the e-PTFE_i (Figure 2(c)) and the e-PTFE_f (Figure 2(d)) were measured by AFM. Due to the distribution of nodes within the fibrillar PTFE network (Figure 2(a)), the histogram of the Young's moduli measured for the raw PTFE material suggest a bimodal distribution of the stiffness (Figure 2(b)). Similar results were obtained for e-PTFE_i (Figure 2(c)) as well as for e-PTFE_f (Figure 2(d)). These bimodal distributions of the respective histograms are mirroring the heterogeneity of the biomaterial. According to the Kruskal-Wallis one-way analysis on ranks, there were no statistical differences $(p=0.484)$ between the Young's moduli of the three surfaces; i.e. raw PTFE, e-PTFE_i and e-PTFE_f.

Water contact angles decrease from $121^{\circ} \pm 3^{\circ}$ for the e-PTFE to $77^{\circ} \pm 4^{\circ}$ for the e-PTFE_f on the PDA film and to $92^{\circ} \pm 10^{\circ}$ (Figure 3 ) for the e-PTFE_i, regardless of the side (Figure 3). This last measure is in agreement with the fact that there is more PDA material at the surface of the e-PTFE_f sample and also with the changes in film roughness as evidence in Figure 1(b) and Figure 1(c).

The cellular behaviors of primary fibroblasts and WJ-SC on the PDA impregnated biomaterial were assessed by transmission electron microscopy and environmental scanning electron microscopy. TEM observations revealed that fibroblasts exhibiting typical elliptical nuclei were lining the e-PTFE substrate (Figure 4(a) et Figure 5(a)). The abundant endoplasmic reticulum emphasizes their active state (Figure 4(b)). ESEM observations carried out on stem cells cultured onto the e-PTFE_i confirmed the cell spreading at the biomaterial's surface (Figure 4(a)).

The cellular behavior of primary fibroblasts and WJ-SC was also investigated, by using the same techniques, on the PDA coated biomaterial. These observations revealed a very close contact between the fibroblast layer and the e-PTFE_f (Figure 5(a)). The numerous focal contacts exhibited by the cultured cells emphasize 
cell attachment to the nanoscale PDA coating (Figure 5(a)). When cells were put onto e-PTFE which was imperfectly covered by a PDA layer, one can note that cells grew preferentially over the PDA coated areas and, more interestingly, send cytoplasmic projections toward the PDA coating (Figure 5(b)).

Inflammation is a normal and necessary response to an injury to initiate the

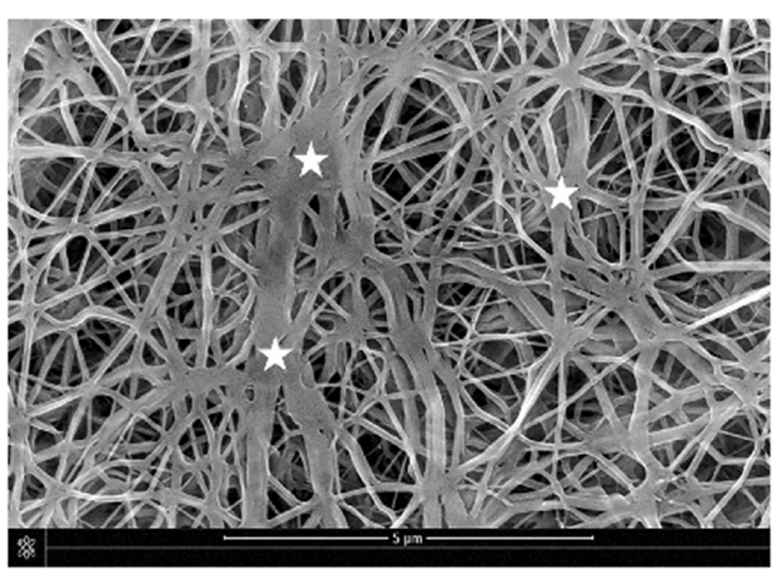

(a)

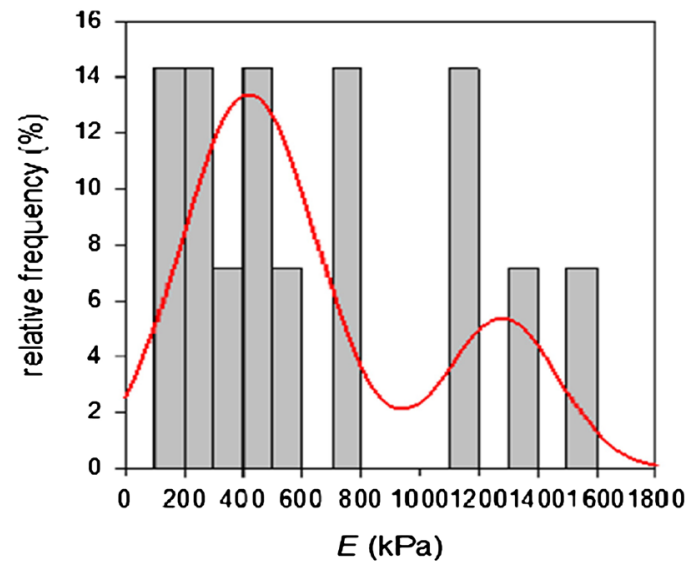

(c)

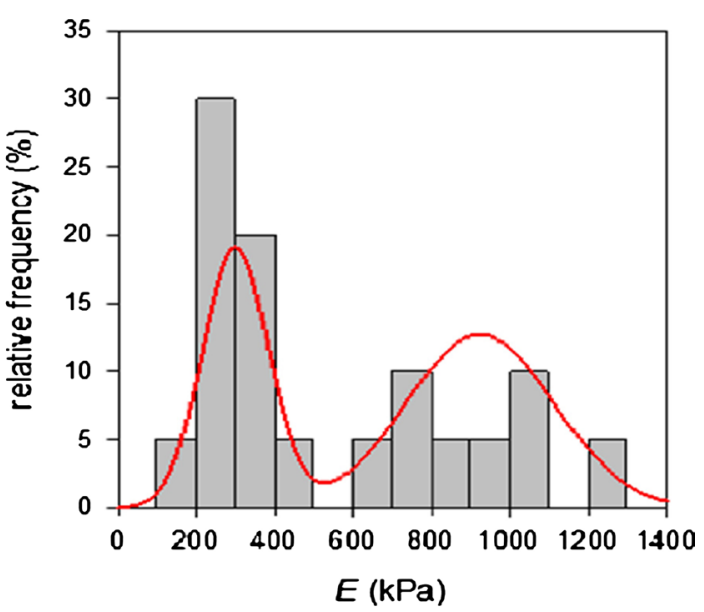

(b)

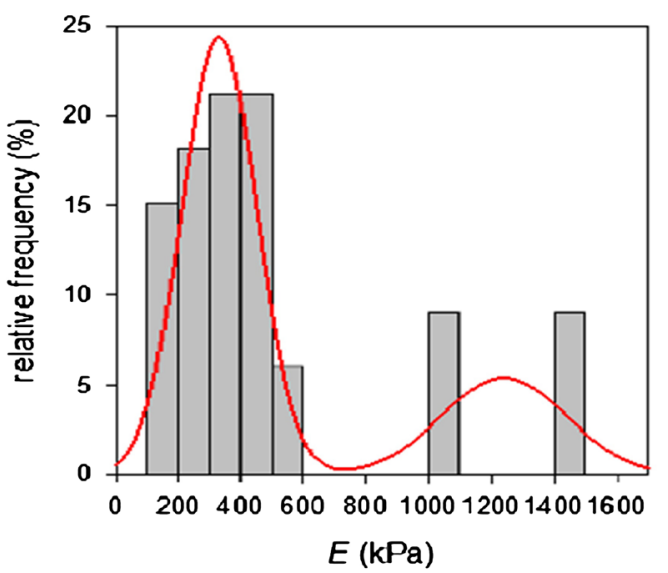

(d)

Figure 2. (a) SEM micrograph showing the fibrillary structure of the e-PTFE material. One can note the distribution of nodes (stars) within the PTFE network. Scale bar $=5 \mu \mathrm{m}$. (b) Histogram of the Young's moduli of the e-PTFE raw material measured with AFM $(n=20)$. It suggests a bimodal distribution of the stiffness mirroring the heterogeneity of the material. (c) Histogram of the Young's moduli of e-PTFE_i measured by AFM ( $n=14)$. It suggests a bimodal distribution of the stiffness mirroring the heterogeneity of the biomaterial. (d) Histogram of the Young's moduli of e-PTFE_f measured by AFM $(n=33)$. It suggests a bimodal distribution of the stiffness mirroring the heterogeneity of the biomaterial.

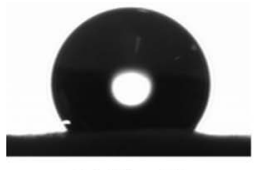

$121^{\circ} \pm 3^{\circ}$

e-PTFE

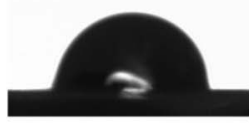

$92^{\circ} \pm 10^{\circ}$

e-PTFE_f

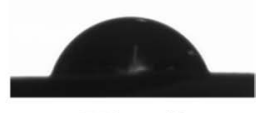

$77^{\circ} \pm 4^{\circ}$

e-PTFE_i

Figure 3. Water contact angles of e-PTFE, e-PTFE_f and e-PTFE_i membranes, at room temperature on the rought side of e-PTFE. 


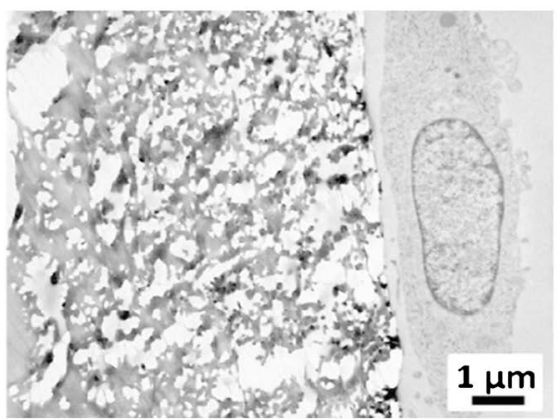

(a)

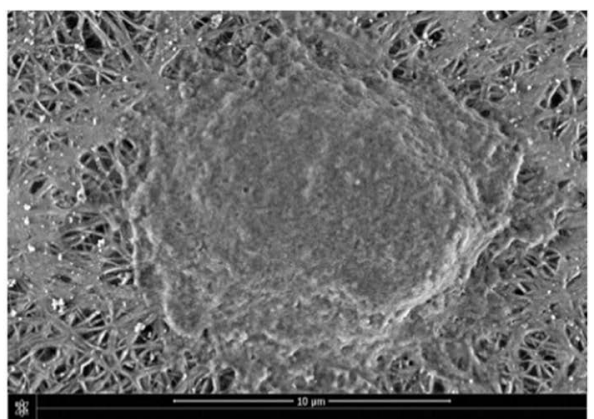

(b)

Figure 4. (a) TEM micrograph showing a primary fibroblast lining e-PTFE_i. Scale bar = $1 \mu \mathrm{m}$; (b) ESEM micrograph showing a Wharton's jelly stem cell spread onto e-PTFE_i. Scale bar $=10 \mu \mathrm{m}$.

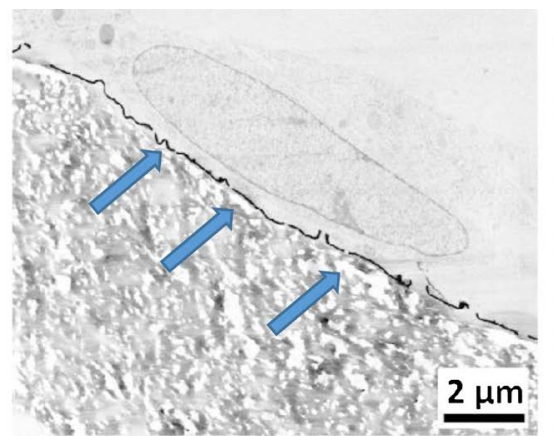

(a)

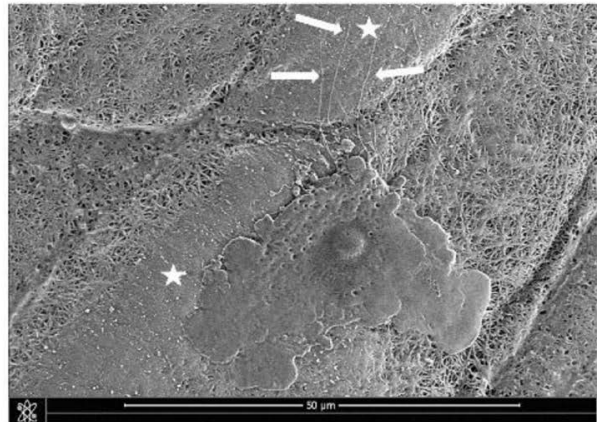

(b)

Figure 5. (a) TEM micrograph showing the growth of primary fibroblasts at the surface of e-PTFE_f. Scale bar $=2 \mu \mathrm{m}$. Numerous focal contacts of the cultured cells are evident at the surface of the nanoscale PDA coating (arrows). (b) ESEM micrograph showing a Wharton's jelly stem cell spread onto e-PTFE_f. One can note that cytoplasmic projections (Arrow) are oriented toward the PDA coated area. Scale bar $=50 \mu \mathrm{m}$.

repair but the equilibrium is really weak [11] and the modified e-PTFE should be checked for this ability to induce inflammation. Murine monocyte/macrophages, namely RAW264.7 cells were used and first, regarding the mitochondrial activity which should reflect the cell viability, we do not find any significant difference between the different conditions (Figure 6), as expected. Nevertheless, the cells observed under a simple microscope reveal a different cell shape in the presence of PDA. From round cells as in control or e-PTFE well, RAW264.7 cells tend to spread with extensions in various directions and vacuoles in well with modified e-PTFE (Figure 7) which look much more like activated cells. To investigate if there is a real inflammatory response, TNF- $\alpha$ production was determined by ELISA. It appears that in presence of e-PTFE_i, RAW264.7 cells produce TNF- $\alpha$ (Figure 8).

\section{Discussion}

In the majority of referral centers, e-PTFE prostheses continue to be favored for the surgical repair of congenital diaphragmatic hernia [1]. Even though collective experience has demonstrated that $\mathrm{CDH}$ outcome is dependent on patient 


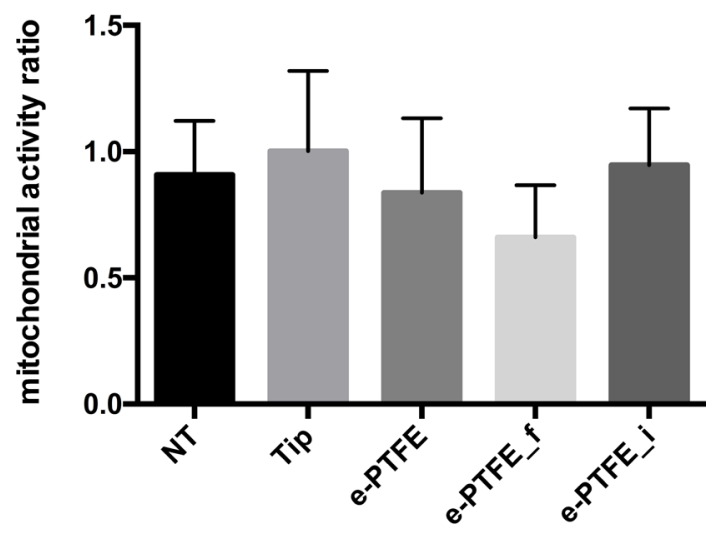

Figure 6. e-PTFE or modified ePTFE with DPA do not seem to affect mitochondrial activity and by extension cell viability. RAW264.7 cells were incubated with e-PTFE or modified ePTFE with DPA during $24 \mathrm{~h}$ before mitochondrial activity was assessed using an MTS test. NT: not treated (i.e.: cells alone); Tip: tool for maintaining e-PTFE at the bottom of wells.
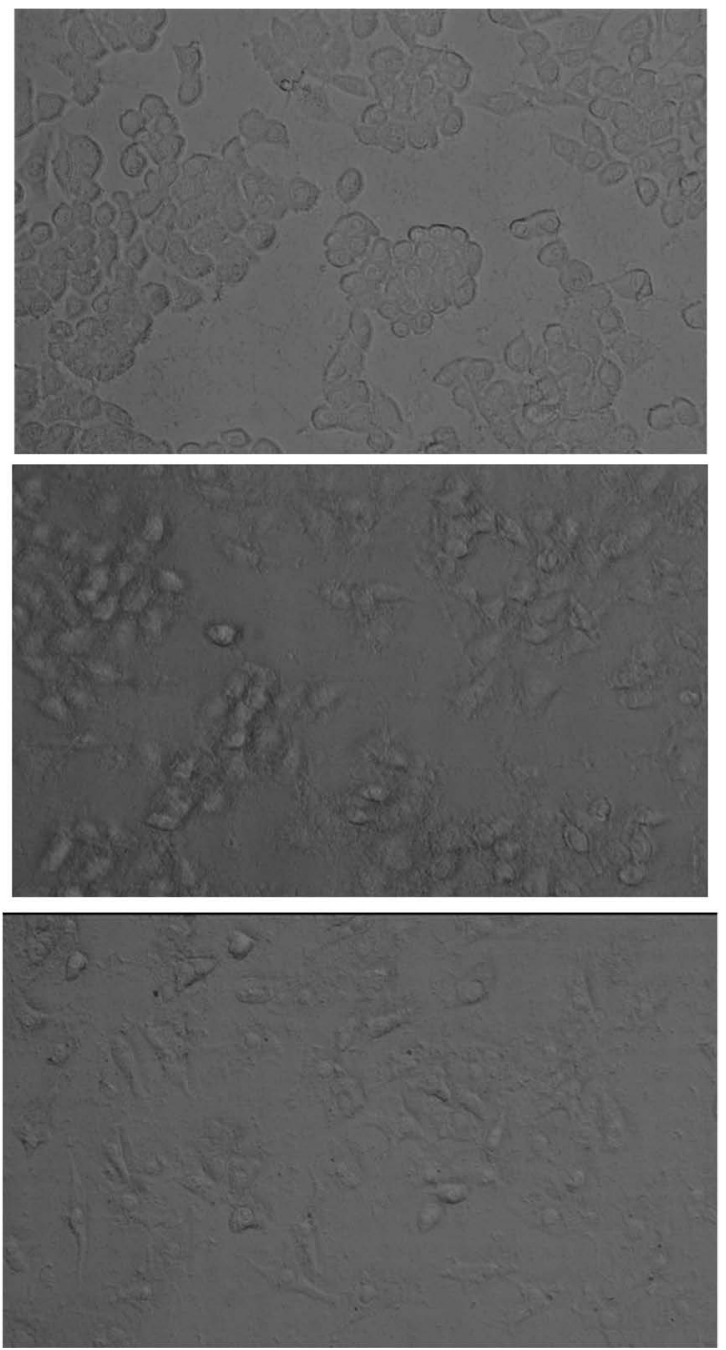

Figure 7. from up to down: Optical microscopy analyses of living cells onto e-PTFE, e-PTFE_f and e-PTFE_i, respectively; $\times 40$. RAW cell shape changes drastically in presence of PDA. 


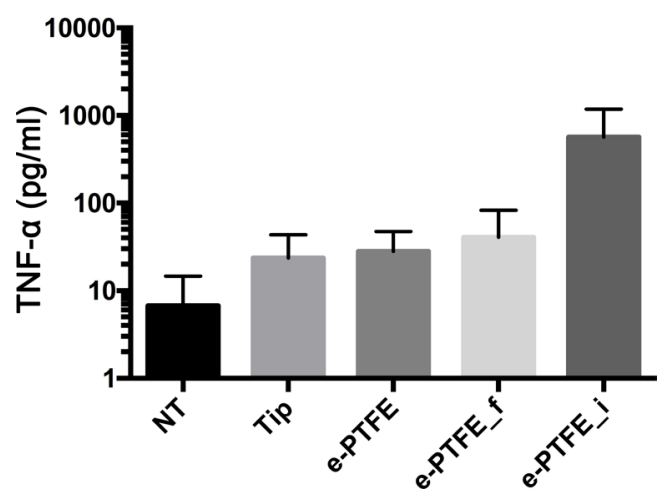

Figure 8. RAW264.7 cells cultured with modified e-PTFE seems to undergo activation as shown by TNF- $\alpha$ production. RAW264.7 cells were incubated with e-PTFE or modified ePTFE with DPA during $24 \mathrm{~h}$ before TNF- $\alpha$ concentration in supernatant was determined by ELISA. NT: not treated (i.e.: cells alone); Tip: tool for maintaining e-PTFE at the bottom of wells.

factors, as hernia side and presence of associated anomalies [12], it also appears that the biological host response is of utmost importance. Indeed, the rapid growth of the child that takes place during infancy and the continuous movements due to breathing subjects the implant to traction forces that can lead to prosthesis detachment [13]. Hence a solid tissular integration of the implanted patch is expected. The periphery of the biomaterial is the best path for cellular and capillary ingrowth. Undoubtedly pores of the implant facilitate the ingrowth of host-derived cells into the prosthesis. That is why the actual used e-PTFE (Figure 1(a)) has a porosity. For instance, it has been demonstrated that the endothelial coverage of PTFE surfaces can be modulated simply by changing the porosity of the graft material [14].

Cellularization of a biomaterial that separates thorax from abdomen is not trivial, as many biomaterials used for tissue engineering applications lack cell-adhesiveness [15]. For this reason, PTFE has to be chemically activated and subsequently modified by grafting specific peptides.

Here we report two functionalization strategies of the e-PTFE material by using PDA which is quite similar to the dihydroxy-phenylalanine found in mussel proteins and then, mussel-inspired chemistry become a hot research focus in biomedical science [16] [17] Some have focused their research on the influence of treatment time with PDA on the property of PTFE-fiberglass [18], we focused our research on two way of impregnation. The first method consists in an impregnation with PDA of the biomaterial. SEM observations revealed that this treatment provides a thickening of the individual PTFE threads, thus causing a decrease of the porosity (Figure 1(b)). A second functionalization technique consists in the deposition of a thin PDA film at only one side of the biomaterial (Figure 1(c)). AFM observations showed that both PDA functionalization methods lead to the coalescence of nano-sized PDA particles (Figure 1(d), Figure 2). When the biomaterial was immersed in a PDA solution, the fibrils of the porous PTFE architecture were decorated with PDA grains. On the other hand, 
the coverage of the implant material with a PDA film, by using the Langmuir-Schaeffer transfer method, disclosed a more or less uniform granular coating. The ultrathin PDA layer, measuring less than $100 \mathrm{~nm}$ in thickness, forms a flat surface onto the porous biomaterial (Figure 2). Tanks to the thin nature of the PDA layer $(\sim 80 \mathrm{~nm})$ the total quantity of active material is kept to a minimum. The possibility to cover solely one side of the PTFE membrane with a PDA nano-layer is surgically spoken of the highest interest, since adhesions have been associated with $\mathrm{CDH}$ repair with prosthetic patches. The Langmuir-Schaeffer technique offers the prospect of producing implants with two sides having different surface properties. Moreover, almost any sheet-like biomaterial could be treated by this way.

As we know that mechanical properties of a substrate are able to influence single and collective cell behaviors [19] [20], it seemed relevant to investigate the potential changes of the Young's moduli inherent to both functionalization methods. SEM micrographs showed that the e-PTFE surface consisted of PTFE strands that intersect, thus forming denser areas. Therefore, the e-PTFE biomaterial surface is actually divided in two different fields, i.e. scattered threads or compact nodes. Colloidal probe AFM measurement confirmed that last observations. The histogram of the Young's moduli of the e-PTFE raw material suggests a bimodal distribution of the stiffness manifesting the described heterogeneity of the material (Figure 3(b)). AFM analyses carried out on the e-PTFE_i also revealed a bimodal distribution of the stiffness, similar to that of the raw biomaterial (Figure 3(c)). Nevertheless, a very slight increase of the stiffness value, due to the PDA treatment, can be mentioned. Surprisingly the deposition of an ultrathin PDA film, although hiding the fibrillar architecture of the PTFE construct, does not alter the bimodal distribution of the stiffness (Figure 3(d)). Accordingly, one can expect that cellular responses will not be impacted by a change of mechanical factors.

Analysis of static contact angles shows that e-PTFE_i is more hydrophilic than e-PTFE_f. Furthermore, both functionalization methods do not induce cell mortality, but the e-PTFE_i condition produces more TNF $\alpha$. This can be explained by the fact that e-PTFE_i contains probably more PDA particules due to the e-PTFE porosity and thus an impregnation of the e-PTFE by the PDA solution. Immune cell shapes change when culture onto e-PTFE, e-PTFE_f or e-PTF_i from a round shape to a more spreading appearance with cytoplasmic prolongations suggesting that there is a cellular activation. This change of the cell shapes might reveal an increasing proinflammatory effect. Finally, we can assume that PDA does not affect cell viability and favors cell development. On the other hand, the immune responses seem to depend on PDA concentration on the material, since the morphological changes are more marked for e-PTFE_i than e-PTFE_f.

In the present study we investigated by TEM and ESEM the behavior of primary fibroblasts and WJ-SC grown onto raw e-PTFE, e-PTFE_f and e-PTFE_i. 
Ultrathin sectioning of the porous e-PTFE with the cellular material on its surface was a challenge, since the implanted material remains very soft compared to the embedding resin [21]. Indeed, some authors performed TEM analysis on PTFE vascular grafts in animals, but they never presented intact interfaces between PTFE and biological material [14] [22]. Even more, recent reports emphasize the difficulty to show complete undamaged interfaces between this biomaterial and the surrounding biological tissues [23]. It seems also noteworthy to mention that the ESEM technique limits potential artefacts that are likely to appear after dehydration and metal coating of biologic specimens.

Thorough analyses of intact interfaces between e-PTFE_i and fibroblasts revealed a close contact of the cell layer with the biomaterial (Figure 4(a), Figure 5(a)). ESEM observations realized in environmental conditions, without metal coating, disclosed a regular spread of cells onto e-PTFE_i (Figure 4(b), Figure 5(b)). TEM micrographs from fibroblasts grown onto the PDA ultrathin film showed similar results (Figure 5(a)). AFM investigations disclosed the synthesis of collagen fibers with typical banding at the PDA-covered biomaterial. However, when cells were seeded on the PDA coating, much more focal contacts were evident. It must also be pointed out that, in the case of PDA-coating defaults, cells grew preferentially onto the PDA covered areas and tended to spread toward the PDA nano-layer (Figure 5(b)). This last result underlines the biological interest of a PDA-coating to favor tissular integration of a PTFE biomaterial. Furthermore, our preliminary results on macrophage cells seemed to in agreement with this positive effect of DPA by inducing "positive" inflammation.

\section{Conclusion}

Present results strongly suggest that PDA functionalization is a smart method to modify the surface of biomaterials. The PDA nano-sized coating described here offers an easy way to modify solely one side of patches used for $\mathrm{CDH}$ repair. Observed cellular behaviors emphasize the interest of the presented method to optimize surgical implantation and maximize long-term graft function. Since we now have a convenient mean of functionalizing a single face of a PTFE prosthesis, it seems highly interesting to explore two additional ways to understand and improve the integration of this kind of biomaterials. First, it seems appropriate to complete this chemical functionalization of the material with a biological coating that would further promote, and even guide, tissue integration. Secondly, it would be equally important to study biological responses on substrates subjected to mechanical stress, which is closer to clinical realities.

\section{Author Contributions}

Conceptualization: Isabelle Talon, Anne Schneider, Vincent Ball, Joseph Hemmerlé, Benoit Frisch; Data curation: Isabelle Talon, Cendrine Seguin, Anne Schneider; Formal analysis: Anne Schneider, Isabelle Talon and Bernard Senger; Investigation: Isabelle Talon, Anne Schneider, Cendrine Seguin and Eric Ma- 
thieu; Methodology: Isabelle Talon, Anne Schneider, Vincent Ball, Benoit Frisch and Joseph Hemmerlé; Resources: Anne Schneider and Eric Mathieu; Software: Bernard Senger; Supervision: Joseph Hemmerlé; Validation: Vincent Ball,, Cendrine Seguin, Benoit Frisch and Joseph Hemmerle; Writing-original draft: Isabelle Talon, Anne Schneider; Writing-review \& editing: Isabelle Talon, Cendrine Seguin, Benoit Frisch, Vincent Ball, and Joseph Hemmerlé.

\section{Conflicts of Interest}

The authors declare no conflicts of interest regarding the publication of this paper.

\section{References}

[1] Congenital Diaphragmatic Hernia Study Group (2007) Defect Size Determines Survival in Infants with Congenital Diaphragmatic Hernia. Pediatrics, 120, e651-e657. https://doi.org/10.1542/peds.2006-3040

[2] Discher, D.E., Janmey, P. and Wang, Y.L. (2005) Tissue Cells Feel and Respond to the Stiffness of Their Substrate. Science, 310, 1139-1143.

https://doi.org/10.1126/science.1116995

[3] Orr, A.W., Helmke, B.P., Blackmann, B.R. and Schwartz, M.A. (2006) Mechanisms of Mechanotransduction. Developmental Cell, 10, 11-20. https://doi.org/10.1016/j.devcel.2005.12.006

[4] Kalaba, S., Gerhard, E., Winder, J.S., Pauli, E.M., Haluck, R.S. and Yang, J. (2016) Design Strategies and Applications of Biomaterials and Devices for Hernia Repair. Bioactive Materials, 1, 2-17. https://doi.org/10.1016/j.bioactmat.2016.05.002

[5] Ratner, B.D. (2004) Surface Properties and Surface Characterization of Materials. In: Ratner, B.D., Hoffman, A.S. and Schoen, F.J., Eds., Biomaterials Science: An Introduction to Materials in Medicine, 2nd Edition, Elsevier Academic Press, London, 40-59.

[6] Matthews, B.D., Pratt, B.L., Pollinger, H.S., Backus, C.L., Kercher, K.W., Sing, R.F. and Heniford, B.T. (2003) Assessment of Adhesion Formation to Intra-Abdominal Polypropylene Mesh and Polytetrafluoroethylene Mesh. Journal of Surgical Research, 114, 126-132. https://doi.org/10.1016/S0022-4804(03)00158-6

[7] Liu, Y., Ai, K. and Lu, L. (2014) Polydopamine and Its Derivative Materials: Synthesis and Promising Applications in Energy, Environmental, and Biomedical Fields. Chemical Reviews, 114, 5057-5115. https://doi.org/10.1021/cr400407a

[8] Ponzio, F., Payamyar, P., Schneider, A., Winterhalter, M., Bour, J., Addiego, F., Krafft, M.P., Hemmerle, J. and Ball, V. (2014) Polydopamine Films from the Forgotten Air/Water Interface. The Journal of Physical Chemistry Letters, 5, 3436-3440. https://doi.org/10.1021/jz501842r

[9] Butt, H.J. and Jaschke, M. (1995) Calculation of Thermal Noise in Atomic Force Microscopy. Nanotechnology, 6, 1-7. https://doi.org/10.1088/0957-4484/6/1/001

[10] Hertz, H. (1881) Über die Berührung fester elastischer Körper. Journal für die reine und angewandte Mathematik, 92, 156-171.

[11] Kharraz, Y., Guerra, J., Mann, C.J., Serrano, A.L. and Muñoz-Cánoves, P. (2013) Macrophage Plasticity and the Role of Inflammation in Skeletal Muscle Repair. $\mathrm{Me}$ diators of Inflammation, 2013, Article ID: 491497.

https://doi.org/10.1155/2013/491497 
[12] Fisher, J.C., Haley, M.J., Ruiz-Elizalde, A., Stolar, C.J. and Arkovitz, M.S. (2009) Multivariate Model for Predicting Recurrence in Congenital Diaphragmatic Hernia. Journal of Pediatric Surgery, 44, 1173-1179. https://doi.org/10.1016/j.jpedsurg.2009.02.043

[13] Fauza, D.O. (2014) Tissue Engineering in Congenital Diaphragmatic Hernia. Seminars in Pediatric Surgery, 23, 135-140. https://doi.org/10.1053/j.sempedsurg.2014.04.004

[14] Clowes, A.W., Kirkman, T.R. and Reidy, M.A. (1986) Mechanisms of Arterial Graft Healing: Rapid Transmural Capillary Ingrowth Provides a Source of Intimal Endothelium and Smooth Muscle in Porous PTFE Prostheses. The American Journal of Pathology, 123, 220-230.

[15] Gabriel, M., Niederer, K., Becker, M., Raynaud, C.M., Vahl, C.F. and Frey, H. (2016) Tailoring Novel PTFE Surface Properties: Promoting Cell Adhesion and Antifouling Properties via a Wet Chemical Approach. Bioconjugate Chemistry, 27, 1216-1221. https://doi.org/10.1021/acs.bioconjchem.6b00047

[16] Yang, H.C., Waldman, R.Z., Wu, M.B., Hou, J., Chen, L., Darling, S.B. and Xu Z.K. (2018) Dopamine: Just the Right Medicine for Membranes. Advanced Functional Materials, 28, Article ID: 1705327. https://doi.org/10.1002/adfm.201705327

[17] Yang, H.C., Luo, J.Q., Lv, Y., Shen, P. and Xu, Z.K. (2015) Surface Engineering of Polymer Membranes via Mussel-Inspired Chemistry. Journal of Membrane Science, 483, 42-59. https://doi.org/10.1016/j.memsci.2015.02.027

[18] Jiang, Z., Jiang, L., Jia, H., Zhou, Y., Ma, J. and Chen, S. (2018) Modification of Polytetrafluoroethylene-Fiberglass Composite Film Using Polydopamine Deposition with Improved Hydrophilicity. Fibers and Polymers, 19, 1760-1766.

https://doi.org/10.1007/s12221-018-8114-2

[19] Engler, A.J., Sen, S., Sweeney, H.L. and Discher, D.E. (2006) Matrix Elasticity Directs Stem Cell Lineage Specification. Cell, 126, 677-689. https://doi.org/10.1016/j.cell.2006.06.044

[20] Breuls, R.G., Jiya, T.U. and Smit, T.H. (2008) Scaffold Stiffness Influences Cell Behavior: Opportunities for Skeletal Tissue Engineering. The Open Orthopaedics Journal, 2, 103-109. https://doi.org/10.2174/1874325000802010103

[21] Schneider, A., Talon, I., Mathieu, E., Schaaf, P., Becmeur, F. and Hemmerlé, J. (2017) New Insight in the Biological Integration of Polytetrafluoroethylene from an Explant Used for Diaphragm Repair. Journal of Biomaterials Applications, 31, 844-850. https://doi.org/10.1177/0885328216676757

[22] Mellander, S., Fogelstrand, P., Enocson, K., Johansson, B.R. and Mattsson, E. (2005) Healing of PTFE Grafts in a Pig Model Recruit Neointimal Cells from Different Sources and Do Not Endothelialize. European Journal of Vascular and Endovascular Surgery, 30, 63-70. https://doi.org/10.1016/j.ejvs.2005.02.051

[23] Bax, D.V., Wang, Y., Li, Z., Maitz, P.K., McKenzie, D.R., Bilek, M.M. and Weiss, A.S. (2011) Binding of the Cell Adhesive Protein Tropoelastin to the PTFE through Plasma Immersion Ion Implantation Treatment. Biomaterials, 32, 5100-5111. https://doi.org/10.1016/j.biomaterials.2011.03.079 